Aus der Chirurgischen Klinik der Universität in Heidelberg.

(Direktor: Geheimrat Prof. Dr. V. Czerny, Exzellenz.)

\title{
Erfahrungen über die therapeutische Wirkung
} der Radiumstrahlen.

Von Dr. Richard Werner und Dr. Georg Hirschel, Assistenten der Klinik.

Trotz der kurzen Zeit, die seit den ersten Versuchen über die therapeutische Wirkung der Radiumstrahlen verflossen ist, liegen bereits zahlreiche Berichte über die Erfolge dieser Methode vor. Bei kritischer Durchsicht der einschlägigen Publikationen fällt vor allem eine ziemlich beträchtliche Divergenz der Anschauungen über den Heileffekt des Verfahrens auf. Die meisten rühmen die günstige Beeinflussung verschiedener Affektionen, insbesondere der Epitheliome und Sarkome, der Angiome und des Lupus der Haut, andere hingegen verhalten sich den momentanen Erfolgen gegenüber recht skeptisch und warnen vor den Spätfolgen, welche nach stärkeren Bestrahlungen (Ueberexposition) auftreten sollen. Wenn nun auch die Zahl der günstigen Stimmen überwiegt, so kann man doch von einem Kampfe der Meinungen sprechen, der noch weit von einer definitiven Entscheidung entfernt ist, nicht allein, was den Wert des Radiums als Heilmittel überhaupt, sondern auch was die Indikationen für die Anwendung desselben betrifft. Daher mag jede Mitteilung neuer Beobachtungen derzeit noch als ein willkommener Beitrag zum Ausbau dieses modernsten Zweiges der Heilkunde erscheinen und dürften die im folgenden zusammengestellten Resultate einiger einschlägiger Versuche nicht ohne Interesse sein.

Durch die Güte des Herrn Prof. Petersen, ${ }^{4}$ ) dem wir hiermit unseren verbindlichsten Dank aussprechen, standen uns drei Radiumbromidkapseln mit je $10 \mathrm{mg}$ dieser Substanz zur Verfügung. Wir haben mit denselben an 22 Patienten unserer Klinik 33 verschieden lokalisierte Affektionen behandelt, wobei nur jene Fälle in Betracht gezogen sind, welche zwischen Ende

4) cf. dessen Bericht: Therapeutische Versuche mit Radium. Münchener medizinische Wochenschrift 1904, No.21. Sitzung der medizinischen Sektion des Naturhistorischmedizinischen Vereins in Heidelberg atn 9. Februat 1904. 
August 1903 und Ende März 1904 bestrahlt wurden, so daß die letzte Sitzung mindestens fünf, bei manchen Patienten aber schon über zehn Monate zurückliegt. Wenn nun auch diese Beobachtungszeit noch nicht hinreicht, um mit Sicherheit alle Spätfolgen der Radiumapplikation ausschließen zu können, da ja bekannt ist, daf solche, bei den Röntgenstrahlen wenigstens, mitunter erst nach 12-15 Monaten auftreten, so genügt die Frist doch immerhin, um sich ein Urteil über den therapeutischen und kosmetischen Effekt der Methode zu bilden und mit jenem anderer Verfahren $\mathrm{zu}$ vergleichen.

Zur Behandlung gelangten: Karzinom fünfmal, Melanosarkommetastasen einmal (an 11 verschiedenen Stellen), Angiom fünfmal, Lupus siebenmal, Naevus zweimal (darunter ein Fall mit zwei Lokalisationen), ein Narbenkeloid und ein Ulcus tub. linguae. Die Kapsel wurde stets unter Einschlub in einem Gummifingerling mittels Heftplasterstreifen fixiert oder mit der Hand aufgedrückt.

Ueber die Dauer und Zahl der Einzelsitzungen, die Reaktion auf dieselben und den Enderfolg ist folgendes zu berichten:

\section{A. Maligne Tumoren.}

F all 1. 42 jährige Frau, Drüsenkarzinom der Mamma, ausgedebnte, nicht exulzerierte Infiltrationen beider Brüste mit zahlreichen metastatischen Knoten in der Umgebung; geringfügige Schwellung der Achseldrüsen. Im Verlaufe von 24 Tagen werden 32 verschiedene Stellen fünf- bis sechsmal halbstündigen Bestrahlungen unterzogen. Nach der zweiten Bestrahlung (vier Tage nach der ersten) Erytheme, nach weiteren zwei Tagen zirkumskripte Nekrosen der Hautoberfläche teils mit, teils ohne Blasenbildung. Es entstehen kleine Substanzverluste, doch läßt sich makroskopisch auch nach Beendigung der ganzen Behandlung weder Erweichung noch Schwund des Infiltrates konstatieren. Vier Wochen nach der letzten Bestrahlung Tod an Lungenmetastasen

Die histologische Untersuchung ergibt: $1 / 4-1 / 2 \mathrm{~cm}$ tiefe erbsengroße Substanzverluste, zum Teil ausoefüllt mit nekrotischen Epithelmassen, darunter hämorrhagische Entzündung des Coriuns; einzelne Karzinomnester in letzterem im Zerfall begriffen oder bis auf kleine Spuren verschwunden. Die Hauptherde in der Tiefe von mehr als $1 \mathrm{~cm}$ unter der Cutisoberfläche histologisch intakt.

Fall 2. 68 jähriger Mann; großes, jauchendes Karzinom der rechten Gesichtshälfte, Auge, Lider, Wange und Nase ersetzend Der größte, dem rechten Unterlide entsprechende Geschwulstknoten wird zwei Wochen hindurch täglich an zwei verschiedenen Stellen je eine halbe Stunde lang bestrahlt. Vom achten Tage an Rötung der Applikationsstellen, zunehmende Schmerzhaftigkeit, weshalb der sehr empfindliche Patient schließlich nach 14 Tagen die weitere Behandlung verweigert. Nach vier Wochen keinerlei Aenderung bemerkbar. Nach drei Monaten rascher, jauchiger Zerfall des ganzen Tumors (sicher unabhängig von der Radiumwirkung).

Fall 3. 59 jähriger Mann; talergroßes, kraterförmig exulzeriertes Epitheliom der linken Schläfengegend. Bestrahlung 16 Tage hindurch an drei Stellen je eine halbe Stunde. Nach fünf Tagen Rötung, nach einer Woche lokale Nekrose, Nach 14 Tagen Abflachung des Epithelioms am hinteren Rande, dagegen rascheres Fortschreiten am vorderen gegen den Orbitalrand zu. Daher Exzision am 17. Tage.

Fall 4. 52jähriger Mann. Markstückgroßes Unterlippenkarzinom (Rezidir nach keilförmiger Exzision vor drei Vierteljahren), nicht exulzeriert, ohne nachweisbare Drüsenschwellungen. Eine halbstündige Bestrahlung an vier Stellen 12 Tage fortoesetzt. Am Ende der Behandlung deutliche Hyperämie und lokale Exulzeration; nach vier Wochen Abflachung des Karzinoms, aber Drüsenmetastasen; daher Exstirpation des Karzinoms und der Drüsen.

Fall 5. 58jähriger Mann. Epitheliom am rechten inneren Augenwinkel auf beide Lider übergreifend; im Zentrum stark exulzeriert. Acht Tage hindurch täglich an drei Stellen je 30-40 Minuten bestrahlt. Außer Hyperämie keine deutliche Wirkung. Wegen Progredienz an den Lidern am neunten Tage exstirpiert, Defekt durch Plastik gedeckt.

Fall 6. 49 jähriger Mann. Melanosarkomrezidiv. Vor fünf Jahren Melanosarkom am linken Malleolus internus vor 1 $\frac{1}{2}$ Jahren amputatio cruris, da trotz mehrfacher lokaler Exstirpation und Thermokauterisation der Tumor stets rezidiviert war.

Vor 11/2 Jahren Drüsenmetastasen in der linken Leistenbeuge, die exstirpiert wurden, doch bald darauf neuerliches Rezidiv in der Operationsnarbe, später rasch den ganzen Oberschenkel und den alten Amputationsstumpf mit Metastasen von der verschiedensten Form und Größe übersäend; seit zirka drei Wochen einige kleine Knötchın an den Armen, am Rücken und am Kopfe. 11 verschiedene Knoten wurden systematisch fast drei Monate hindurch bestrahlt; an manchen Stellen blieb die Kapsel bis zu 12 Stunden ununterbrochen liegen; wiederholt wurde eine vier- bis sechsstündige Applikation versucht. Ein Punkt auf der Kuppe eines großen Knotens am Oberschenkel war im ganzen ungefähr 100 Stunden der Radiumwirkung ausgesetzt. Trotzdem bestand der ganze Effekt nur in zirkumskripten bis markstückgroßen Hautnekrosen, die wohl etwas tiefer wurden, als dies sonst zu beobachten ist, aber keineswegs zu ausgedehnterer Zerstörung des Gewebes führten. Von einer spezifischen Beeinflussung (Erweichung, Resorption) der Tumormassen war nichts zu bemerken. Später wurden auch Röntgenstrahlen ohne Erfolg angewendet. Zwei stark melanotische, etwa kirschkerngroße Knötchen, die kugelig über die Haut prominierten, verschwanden einige Wochen nach mehrstündiger $\mathrm{Be}$ strahlung; hier scheint außer der lokal nekrotisierenden auch eine spezifische Wirkung auf das Tumorgewebe vorzuliegen.

Bei den hier mitgeteilten sechs Fällen von bösartigen Neubildungen war somit die Radiumbehandlung erfolglos. Dies steht jedoch zu den vielfach gemeldeten Heilungen von "Karzinomen" und "Sarkomen" nur scheinbar im Widerspruch. Es handelte sich bei den günstig beeinfluhten inalignen Affektionen wohl immer um ganz oberflächliche Epitheliome oder Hautsarkome, welche die sowohl von Exner wie neuerdings auch von Apolant auf zirka $1 \mathrm{~cm}$ geschätzte Wirkungstiefe der Radiumstrahlen nicht überschritten, wenn sie auch infolge ihrer Flächenausdehnung inoperabel sein mochten. Wir konnten sowohl am Karzinome als auch beim Tierexperimente an der Haut von Meerschweinchen histologisch nachweisen, daß die Veränderungen sich stets auf die angegebene Zone beschränkten.

Daß Karzinomzellen auch dann, wenn sie außerhalb der direkt nekrotisierten Hautpartie liegen, durch die Radiumstrahlen zerstört werden können, war auch bei uns aus einigen mikroskopischen Präparaten zu ersehen; doch scheint der Umfang, in welchem dies der Fall ist, je nach der Art des Tumors zu variieren und damit auch die spezifische elektive Wirkung der Behandlung großen Schwankungen zu unterliegen.

Aus unseren Erfahrungen lassen sich zwar keine Indikationen für, doch gewisse Kontraindikationen gegen die Anwendung des Radiums ableiten, und $\mathrm{zwar}$ dürfte letzteres $\mathrm{zu}$ verwerfen sein, wenn ein weiteres Fortschreiten des Prozesses die operative Entfernung selbst oder wenigstens den Ersatz des Defektes, der durch sie entsteht, erschwert, oder wenn es sich um erfahrungsgemäß leicht Drüsenmetastasen verursachende Geschwulstformen handelt. In beiden Fällen dürfte unbedingt die schnelle Entfernung des Tumors durch das Messer vorzuziehen sein, da bei der Unsicherheit des Erfolges der Radiumbehandlung zum mindesten durch nutzlose Verzögerung Schaden erwachsen kann.

$\mathrm{Ob}$ eine unvollkommene Zerstörung des Tumors durch die Bestrahlung nicht etwa den Rest desselben zul verstärkter Wucherung reizt, wie man dies nach unseren Erfahrungen fast vermuten möchte, wird erst an der Hand eines großen Materials entschieden werden können.

Da wir in Uebereinstimmung mit Exner die Behandlung operabler Karzinome mit Radium nicht empfehlen können, bei inoperablen aber die Chancen der Heilung - oberflächliche Applikation vorausgesetzt - schon bei mäßigem Tiefenwachstume recht geringe werden, so scheint die Radiumtherapie für bösartige Neubildungen nicht die anfangs erhoffte Bedeutung gewinnen zu sollen, falls nicht die intratumerale Anwendung in Form von Sonden oder von Injektionen der gelösten Emanation bessere Resultate erzielt.

\section{B. Gutartige Neubildungen.}

a) Angiome.

Fall 1. 20jähriges Mädchen. Haselnußgroßes Angiom der Unterlippe, durch die ganze Dicke derselben reichend, sowohl von der äußeren Haut als auch von der Schleimhaut deutlich sichtbar und fühlbar. Erst von der Schleimhautseite her zweimal je fünf Minuten bestrahlt, dann von der Außenseite her viermal je 20-30 Minuten jeden zweiten Tag. Nach dem Auftreten einer starken Rötung ausgesetzt. Vier Tage später Beginn der Exulzeration an der Haut, ganz flach, Pfenniggröße erreichend. Nach vier Wochen völlige Benarbung, Haut blaß, pigmentlos, mit zartem bläulichen Schimmer. Angiom nicht mehr nachweisbar. Seither keine Veränderung.

Fall 2. Drei Monate altes Mädchen. Markstückgroßes Angiom am Hinterhaupte, achtmal jeden zweiten Tag an je drei Stellen 15 bis 30 Minuten lang bestrahlt; vom achten Tage nach dem Aussetzen der Bestrahlung an plötzlich rapid um sich greifende Exulzeration, die bis anf das Periost geht. Benarbung erst nach sechs Wochen beendet. Die Stelle ist glatt, haarlos, weiß glänzend mit zartem rötlichen Schimmer. Die Haut daselbst straff gespannt, aber gegen den Knochen verschieblich. Angiom verschwunden. Bisher -- nach fünf Monaten kein Rezidiv. 
Fall 3. Acht Monate altes Mädchen. Talergroßes Angiom der linken Supraorbitalgegend, auf das Oberlid übergreifend. 24 Tage lang Bestrahlung anfangs von drei Stellen jeden zweiten Tag fünf Minuten lang, später fünf Stellen zehn Minuten lang, schließlich 16 Stellen 15 Minuten lang. Das Oberlid wird wegen der Gefahr für das Auge nicht bestrahlt, das Angiom daselbst thermokauterisiert. Vom Ende der Behandlung an starke Exulzeration, die nach drei Wochen benarbt ist. Haut weiß, mit rosigem Schimmer, glatt, verschieblich. Vom Angiom nichts mehr zu sehen. Trotz anscheinend gründlicher Beseitigung desselben Rezidiv nach acht Wochen. (Brieflicher Bericht.)

F all 4. 17jähriges Mädchen. Markstückgroßes Angiom der rechten Wange. Sechsmalige Bestrahlung an jedem zweiten Tage $15 \mathrm{Mi}$ nuten lang. Geringfügige Exulzeration, die nach zirka 14 Tagen glatt benarbt ist. Auffallend blasse, pigmentlose Haut. Angiom spurlos verschwunden. Keine weitere Nachricht zu erhalten gewesen.

Fall 5. 25jähriger Mann. Angiom am Rücken, fast talergroß, deutlich prominierend. Behandlung zwei Wochen hindurch an vier bis fünf Stellen jeden zweiten Tag, 10-20 Minuten lang. Geringfügige Exulzeration, vollkommene Abflachung, doch ist die Narbe noch ziemlich stark gerötet. Aus äußeren Gründen keine weitere Behandlung. Später vollkommenes Abblassen, fast normale Färbung. Angiom verschwunden; seit acht Monaten kein Rezidiv.

\section{b) Naevi.}

Fall 1. 23jähriger Mann. Handtellergroßer, stark (über $1 / 2 \mathrm{~cm}$ ) prominierender, rotbraun gefärbter Naevus verrucosus am rechten Vorderarm, ein zweiter, ebenso großer, von gleicher Beschaffenheit am linken Unterschenkel desselben Patienten. Im Verlaufe von 46 Tagen am Vorderarm 30 Bestrahlungen, jedesmal vier bis fünf Stellen, anfangs zehn Minuten lang, später bis zu einer halben Stunde steigend, im Verlaufe von 17 Tagen 14 BestrahIungen am Unterschenkel, jedesmal vier bis fünf Stellen $1 / 2-3 / 4$ Stunde lang. Der Effekt war an beiden Stellen ein qualitativ gleichartiger, aber quantitativ verschiedener. Am Arm in der zweiten Woche Rötung, dann (in der dritten Woche) ganz oberflächliche Exulzeration; deutliche Abflachung der bestrahlten Partien, auch in der Umgebung, wo keine Epidermisnekrose nachweisbar ist. Nach Beendigung der Behandlung rasche Benarbung, Haut imZentrum blaß, an der Peripherie braun pigmentiert. Am Unterschenkel sowohl Exulzeration, als auch Abflachung geringer. Während am Arm einige Stellen ganz in das Niveau der übrigen Cutis gesunken sind, ist am Unterschenkel eine vollkommene Ausgleichung nicht erzielt worden. Weitere Behandlung aus äußeren Gründen abgebrochen. Nach sieben Monaten erscheint der Zustand nicht wesentlich verändert. Anscheinend hat die Abflachung noch etwas zugenommen und die Randpigmentierung sich vermindert.

Fall 2. 28jähriger Mann; handflächengroßer, flacher, ganz schwarzbrauner Naevus pigmentosus am Hals. Neun Tage hindurch Bestrahlung an fünf bis acht Stellen je eine Viertelstunde lang. Am Ende der Behandlung Exulzeration fast der Hälfte der Naevusfläche. Nach 14 Tagen vollkommen benarbt, im Zentrum blaf, pigmentlos, nach der Peripherie allmählich zunehmende Pigmentierung, an einzelnen Stellen sogar deutliche Vermehrung der letzteren. Weitere Behandlung aus äußeren Gründen abgebrochen.

\section{c) Narbenkeloid.}

25jähriger Mann; Keloid am rechten Handrücken, $2 \mathrm{~cm}$ lang, $1 \mathrm{~cm}$ breit, $1 / 2 \mathrm{~cm}$ hoch, nach einer Inzision vor neun Monaten entstanden. Einmalige Bestrahlung am proximalen Ende 12 Stunden hindurch, am distalen Pole je zwei Stunden lang an drei aufeinanderfolgenden Tagen. Nach zwei Tagen starke Rötung, vom dritten Tage an Ex. ulzeration mit Blasenbildung. Nach zwei Wochen vollständige Benarbung im Niveau der Haut. Cutis glatt, blaß, verschieblich; keine Spur von Keloidbildung vorhanden. Nach sieben Monaten noch unverändert.

\section{Tuberkulide.}

Fall 1. 60 jährige Frau; symmetrischer Lupus vulgaris der oberen Nasenhälfte, schmetterlingsflügelfơrmig sich auf beide Wangen ausbreitend, auf beide Augenwinkel und in Talergröße auch auf die Stirne übergreifend. Außerdem drei isolierte Knötchen in der Nähe der Nasenspitze. Ein Teil des Lupus wird nur einmal an jeder Stelle zwei bis drei Stunden lang, ein anderer neun Tage hindurch jeden zweiten Tag eine halbe Stunde bestrahlt; die drei isolierten Knötchen nur einmal eine halbe Stunde lang. Es zeigt sich eine bemerkenswerte Differenz in der Reaktion der verschieden behandelten Stellen. Die drei Stunden bestrahlten wiesen nach drei bis vier Tagen Zerfall der Lupusknötchen, nach sechs bis sieben 'Tagen Ulzeration der benachbarten Haut auf, an den fünfmal je eine halbe Stunde behandelten Punkten zerfielen die Knötchen fast gleichzeitig mit der angrenzenden, makroskopisch normalen Epidermis. Die drei einzelnen Knötchen bildeten sich ohne Ulzeration zurück und hinterließen punktförmige Narben. Die Geschwüre, welche sich an den übrigen Teilen des Lupus gebildet hatten; heilten nach vier bis sechs Wochen unter Zurücklassung schöner, glatter, weißer, pigmentloser Narben aus.

Während der Behandlung entstand, obwohl die Bindehaut nie direkt bestrahlt worden war, an beiden Augen eine ziemlich beträchtliche Conjunctivitis, an welche sich am linken Auge ein Ulcus corneae anschloß. Die Ausheilung dauerte fast ebensolange wie jene der Ulzerationen auf der Haut. Auf der Hornhaut blieb eine leichte Trübung zurück, die aber - dank ihrer Lage - den Visus nicht beeinträchtigte. (Patientin hatte an beiden Augen schon wiederholt Conjunctivitis eczematosa durchgemacht und schon mehrere Ulcera corneae gehabt.)

Der Dauererfolg ist bisher (nach sechs Monaten) ein durchaus befriedigender; nur an den nicht bestrahlten Augenwinkeln sind noch Knötchen vorhanden; die Benarbung ist eine glatte, kosmetisch sehr günstige.

Fall 2. 23 jährige Frau. Zum Teil exulzerierter Lupus der Nase mit symmetrischem, schmetterlingsflügelförmigem Uebergreifen auf beide Wangen. Beide Nasenflügel teilweise zerstört. Jede Stelle einmal drei Stunden bestrahlt; nach vier Tagen Behandlung beendet. Vom sechsten Tage an rascher Zerfall aller Knötchen, oberflächliche Nekrose der benachbarten Epidermispartien. Nach drei Wochen vollkommene Benarbung. Haut glänzend weiß, verschieblich.

Nach sieben Monaten rezidivfrei, Narbe von fast normaler Hautfarbe. Kosmetischer Erfolg ideal.

Fall 3. 72 jährige Frau. Sehr ausgedehnter, teils verruköser, teils exulzerierter Lupus, die ganze Nase, das mittlere Drittel der Stirn sowie umfangreiche Partien beider Wangen umfassend, mit bedeutenden Defekten an beiden Nasenflügeln. Im Zeitraume von acht Tagen wird die eine Hälfte in der Weise behandelt, daß jede Stelle dreimal je dreiviertel Stunden bestrahlt, der Rest aber an jedem Punkt drei Stunden belichtet wird.

Nach der letzteren Methode wird abermals erst ein Zerfall der Knötchen, dann der benachbarten Epidermis erzielt, während bei fraktionierter Dosierung eine zeitliche Differenz nicht zu konstatieren ist. Zerfall der gesamten Hautoberfläche des erkrankten Gebietes. Heilung desselben per granulationem.

Fall 4. 51 jähriger Mann. Lupus verrucosus von Pfennigstückgröße am rechten Zeigefinger. Im Verlaufe von 14 Tagen wird jede Stelle etwa acht- bis neunmal, von 10-45 Minuten steigend, bestrahlt. Nach vollständiger Exulzeration (am zehnten Tage) wurde jede Stelle doch noch zweimal belichtet. Nach vier Wochen völlige Heilung mịt glatter, kleiner Narbe.

Fall 5. 58 jährige Frau. Lupus verrucosus der rechten Wange (sehr ausgedehnt). Eine zirkumskripte Partie wird 17 Tage hindurch so behandelt, daß jeder Punkt etwa drei- bis viermal erst 10, dann 15, 20 bis 30 Minuten bestrahlt wird. Aus äußeren Gründen weitere Behandlung abgebrochen. Nach vier Wochen sind - ohne Exulzeration - sämtliche Knoten im behandelten Felde verschwunden; es hat sich daselbst eine glatte, weißglänzende Haut gebildet.

Fall 6. 24 jähriger Mann. Lupus hypertrophicans des Handrückens, markstückgroß, stark prominent. Im Zentrum einmalige Bestrahlung acht Stunden hindurch. Darauf nach fünf bis sechs Tagen zentrale erbsengroße Nekrose, peripher heftige Entzündung ohne Ulzeration. Nach drei Wochen Lupus vollständig geschwunden, kleine, weißglänzende, im Niveau der Haut liegende Narbe, entsprechend der nekrotisch gewesenen Partie. Nach acht Monaten kein Rezidiv; Narbe kaum sichtbar.

Fall 7. 16 jähriger Junge. Lupus hypertrophicans des Fußrückens, überhaselnußgroßer Knoten. In 14 Tagen zehnmal je $20 \mathrm{Mi}$ nuten bestrahlt. Nach Beendigung der Behandlung Nekrose der Kuppe des Knotens. Im Verlaufe von weiteren drei Wochen allmähliche Zurückbildung bis in das Niveau der Haut. Kleine zentrale weiße Narbe. Keine weitere Nachricht erhalten.

Fall 8. 48.jähriger Mann. Ulcus tuberculosum der Zungenschleimhaut (Rezidiv nach Exzision wegen Malignitätsverdacht). Starke Schmerzhaftigkeit, zum Teil neuralgischen Charakters. Nach viermaliger Bestrahlung 15-20 Minuten lang in 48 stündigen Pausen Schmerzen geschwunden, nach zirka 14 Tagen Ulcus geheilt, Stelle nicht mehr zu erkennen.

Im Gegensatze zu den ungünstigen Erfahrungen bei den malignen Affektionen können wir somit die Erfolge bei den gutartigen Neubildungen und Tuberkuliden als sehr befriedigend bezeichnen. Wenn wir bei diesen ersten therapeutischen Versuchen zunächst nicht danach strebten, stets durch Bestrahlung in kleinen fraktionierten Dosen eine Rückbildung der pathologischen $\mathrm{Ge}$ webe ohne Zerstörung der Epidermis zu erzielen; sondern durch relativ lange Expositionsdauer ohne Rücksicht auf die Haut nach möglichst sicherer und kräftiger Wirkung trachteten, so lag dies zum Teil an der Beschaffenheit der Fälle, bei denen durch eine mildere Behandlung keine radikale Heilung zu erwarten zu sein schien, zum Teil aber auch daran, dab wir wünschten, die Art der Benarbung nach radiogenen Ulzerationen selbst studieren zu können, um zu entscheiden, ob nicht eine rasche Methode mit wenigen 
Sitzungen durchführbar wäre. Eine solche erscheint namentlich für die ambulante Behandlung entfernt wohnender Patienten von nicht zu unterschätzender praktischer Bedeutung. Es hat sich nun gezeigt, daß nach einer solchen „forcierten“ Belichtung, Ulzerationen entstehen, welche mehrwöchige Heilungsdauer beanspruchen, dann aber in der Mehrzahl der Fälle zu kosmetisch ganz vortrefflicher Benarbung führen, ferner, daß die elektive das pathologische Gewebe ohne Hautnekrose zur Resorption bringende Wirkung des Radiums sich nicht allein auf die kleine von dieser Substanz bedeckte Fläche beschränkt, sondern, insbesondere in der Tiefe, darüber ziemlich weit hinausreicht, so daf es gelingt, durch energische lange Bestrahlung an verhältnismäßig wenigen Punkten flächenhaft ausgedehnte Affektionen zur Heilung zu bringen. Es entstehen dann nur wenige kleine Hautnekrosen, die nach kurzer Zeit ausheilen. Diese Behandlung entspricht etwa der "Stichelung" mit dem Thermokauter oder mit einem Aetzstifte, wobei ebenfalls nicht die ganze Hautdecke zerstört und dennoch der ganze Herd beeinflußt wird, nur daf beim Radium das Verhältnis ein günstigeres zu sein scheint als bei den anderen genannten Mitteln. Das Ideal wird natürlich stets die Heilung ohne Epidermisnekrose bleiben, allein, wo die radikale Beseitigung auf diese Weise mißlingt oder eine rasche Absolvierung der Radiumkur geboten ist, dürfte es sich empfehlen, die eben geschilderte Methode anzuwenden oder doch wenigstens zu versuchen.

Es muß aber darauf aufmerksam gemacht werden, daf die Ulzerationen, welche nach Abstoßung der nekrotischen Partien entstehen, in manchen Fällen recht schmerzhaft sind, wenn nicht eine absolut reizlose Salbe (am besten Lanolin und Vaselin aa) zur Bedeckung verwendet wird. Dies ist auch nicht ohne Einfluß auf die Benarbung, welche dann am schnellsten und mit der relativ geringsten entzündlichen Reaktion erfolgt.

Im allgemeinen scheint es Regel zu sein, daf, was durch die forcierte Behandlung an Zeit gewonnen wird, durch die Dauer der Nachbehandlung wieder verloren geht; da aber letztere an einem beliebigen Orte durchgeführt werden kann, ist bei der ambulanten Behandlung ein gewisser Vorteil für den Patienten nicht zu leugnen. Man mup nur darauf achten, daß zwischen den nekrotischen Hautpartien unbedingt noch intakte, regenerationsfähige Epidermisinseln bestehen bleiben; vernachlässigt man diese Vorsichtsmabregel, oder ist sie infolge der Art der Erkrankung (z. B. bei allzu dichter Dissemination der Lupusknötchen) unmöglich, dann kommt es zu der von vielen Autoren mit Recht gefürchteten, höchst langwierigen Ulzeration, da die Epidermisierung solcher größeren Flächen, auf denen auch nur eine mangelhafte Granulationsbildung stattfindet, außerordentlich langsam vorwärts schreitet.

Ist schon die Ueberhäutung gewöhnlicher Wunden, sobald diese einen gewissen Umfang überschreiten, ein sehr zeitraubender Vorgang, so gilt dies von den durch Radiumwirkung entstandenen in noch höherem Maße. Wir erfuhren dies bei einem Lupus des Gesichtes (No. 3), bei dem die Epidermisierung erst nach vier Monaten beendet war. Um derartige Mißerfolge zu verhüten, wird man gut tun, jene Formen des Lupus, bei denen eine allzu dichte Aussaat der Effloreszenzen stattgefunden hat, sowie ganz flache Angiome und Naevi sehr vorsichtig mit kleinen, fraktionierten Dosen zu behandeln, hingegen dürfte man bei dem deutlich gruppierten verrukösen sowie beim hypertrophischen und exulzerierten Lupus, endlich bei halbkugelig prominierenden Kavernomen und knotigen Naevis die energische Methode mit gutem Erfolge anwenden können.

Bei Beachtung dieser Regeln wird auch die Radiumtherapie kosmetisch mehr leisten als Aetzung oder Thermokauterisation, selbst als Thierschsche Transplantationen oder plastische Deckung nach Exzision, respektive Excochleation. Selbst die Exzision mit linearer Naht kann, ganz abgesehen von der beschränkten Anwendbarkeit der Methode, in günstigen Fällen in bezug auf die geringe Sichtbarkeit der Narbe übertroffen werden.

Von Dauerheilungen kann man nach sechs bis neun Monaten nicht sprechen, aber es ist immerhin bemerkenswert, daß bisher noch kein Rezidiv aufgetreten ist, abgesehen von einem Angiom der Supraorbitalgegend (No. 3) welches aber, soweit es sich auf das Oberlid erstreckte, zur Schonung des Auges thermokauterisiert worden war. Von welcher Stelle die Neuerkrankung ausging, war nicht festzustellen.

Zum Schlusse sei noch auf zwei interessante Beobachtungen aufmerksam gemacht. Beim Lupus war in mehreren Fällen nach einmaliger selır intensiver (dreistündiger Bestrahlung zu konstatieren, daß die Knötchen früher zerfielen als die Epidermis, während bei annähernd gleich langer Gesamtbestrahlung, aber unter fraktionierter Dosierung diese stärkere spezifische Wirkung nicht zu sehen war. Bei einzelnen isolierten Knötchen (No. 1) gelang tatsächlich die Ausheilung ohne Hautnekrose nach einer einzigen Sitzung. Diese Erfahrungen lassen es als denkbar erscheinen, daß es bei genügender Uebung möglich sein wird, gewisse Affektionen in einer Sitzung hinreichend $\mathrm{zu}$ belichten, ohne die Epidermis zu zerstören, da es kein allgemein giltiges Gesetz zu sein scheint, dall die spezifische Wirkung auf pathologische Gewebe bei Anwendung vieler kleiner Dosen gegenüber der deletären auf die Haut stärker hervortritt als bei einmaliger Dauerexposition. Leider sind wir in bezug auf diese „idealsten“ Erfolge vorläufig noch ganz auf den Zufall angewiesen.

Bèmerkenswert ist ferner das Verhalten pigmentierter Stellen gegenüber der Radiumwirkung, indem die Färbung an schwach bestrahlten Punkten sich verstärkte, erst nach Zerstörung der Zellen schwand und im regenerierten Gewebe sich nicht vorfand, obwohl die Epidermisierung - zum Teil wenigstens - durch pigmentierte Haut besorgt wurde. Ob die Bildung pigmentloser Narben nach Radiumwirkung auch auf stark pigmenthaltigem Gewebe ein allgemeingültiges Gesetz ist, mag dahingestellt bleiben, aber auffallend ist die Tatsache, daß alle Narben nach Radiumnekrosen auffallend blaß, glänzend weiß (nach Angiomen allerdings meist auch etwas bläulich oder rötlich schimmernd) sind. Dagegen ist die Vermehrung des hier nicht hämato-, sondern zellulogenen Pigments an schwächer beeinflußten Stellen sicher ein Beweis dafür, daß Prozesse des Zellenlebens, die bei energischer Bestrahlung gehemmt werden, durch einen geringeren Grad der letzteren gefördert, sozusagen "gereizt" werden können. Es ist dies eine weitere Stütze für den Verdacht, daß eine ungenügende Bestrahlung schaden kann, statt zu nützen.

Analoge Erfahrungen über diese insbesondere bei der $\mathrm{Be}$ handlung maligner Tumoren recht fatale Erscheinung hat auch Herr Dr. Engelken bei Versuchen über die therapeutische Wirkung der Röntgenstrahlen gemacht und darüber im Jahresberichte unserer Klinik für das Jahr 1904 die wichtigsten Daten mitgeteilt. (Beiträge für klinische Chirurgie 1904, Supplement.)

Bei benignen Affektionen läßt sich noch darüber streiten, ob man lieber schonend oder schnell vorgehen soll: es wird dies im wesentlichen von der Beschaffenheit des Einzelfalles und den äußeren Umständen abhängen; bei malignen Erkrankungen aber kann es nur eine Regel geben: entweder radikal bestrahlen oder garnicht! 